\section{Revista Facultad de Ciencias Básicas ISSN 1900-4699}

homepage: http://revistas.unimilitar.edu.co/index.php/rfcb

\title{
EVALUACIÓN DE ÁCIDOS ORGÁNICOS EN BEBIDAS DE FRUTA COMERCIALES POR CROMATOGRAFÍA LÍQUIDA DE ALTA EFICIENCIA
}

\section{ORGANIC ACIDS EVALUATION IN COMMERCIAL FRUIT DRINK BY HIGH PERFORMANCE LIQUID CHROMATOGRAPHY}

\begin{abstract}
Yaned Milena CORREA NAVARRO'* , Juan David RIVERA GIRALDO'
${ }^{1}$ Grupo de Investigación en Estudios Ambientales en Agua y Suelo, Facultad de Ciencias Exactas y Naturales, Universidad de Caldas, sede principal, calle 65 N. ${ }^{\circ} 26-10$, Manizales, Colombia.
\end{abstract}

*Autor corresponsal. E-mail: yaned.correa@ucaldas.edu.co
Historia del artículo

Recibido: Junio 17, 2017

Evaluado: Octubre 10, 2017

Aceptado: Noviembre 20, 2017

Disponible: Diciembre 4, 2017

Resumen |

Los ácidos orgánicos son unas de las moléculas constitutivas de los jugos y juegan un papel importante en la determinación de la autenticidad de estos. Este trabajo presenta un método de separación por cromatografía líquida de alta eficiencia (CLAE) de 6 ácidos orgánicos: ascórbico, cítrico, málico, fórmico, succínico y tartárico. El análisis se llevó a cabo empleando una fase estacionaria reversa (C18), una fase móvil constituida

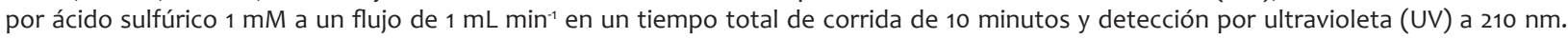
Bajo las condiciones anteriormente descritas se evaluaron 10 muestras comerciales de bebidas de frutas y se encontraron como únicos ácidos el cítrico y el ascórbico, con intervalos de concentraciones de 375,3-5073,7 mg L-11 y 28,4-749,0 mg L-11, respectivamente. La metodología empleada fue simple y apropiada para la separación y la cuantificación rápida, precisa (RSD inferiores al $5 \%$ ), sensible y simultánea de los ácidos mayoritarios en los jugos evaluados. Las curvas de calibración para los ácidos cítrico y ascórbico presentaron excelente linealidad y un alto coeficiente de determinación $\left(r^{2}>0,99\right)$ en los intervalos de concentración empleados.

Palabras clave: análisis cromatográfico, calidad de bebidas, productos naturales, validación.

Abstract

Organic acids are one of the major constituent molecules of fruit juice and play an important role in determining its authenticity. This paper presents a high-performance liquid chromatographic (HPLC) separation method for six organic acids, namely, ascorbic, citric, malic, formic, succinic and tartaric acids. The analysis was performed using a reverse stationary phase (C18), a mobile phase made up of $1 \mathrm{mM} \mathrm{H}_{2} \mathrm{SO}_{4}$ at a flow rate of $1 \mathrm{~mL} \mathrm{~min}^{-1}$, with total run time of 10 minutes and ultraviolet (UV) detection at $210 \mathrm{~nm}$. Under these conditions, ten samples of commercial fruit drinks were tested, and the only acids found were citric and ascorbic acids at concentration ranges of 375.3 to $5073.7 \mathrm{mg} \mathrm{L}^{-1}$ and 28.4 to $749.0 \mathrm{mg} \mathrm{L}^{-1}$, respectively. The method used was simple and appropriate for the fast, accurate (RSD less than $5 \%$ ), sensitive and simultaneous separation and quantification of major acids in the fruit drinks tested. Calibration curves for citric and ascorbic acids showed excellent linearity and a high coefficient of determination $\left(r^{2}>0,99\right)$ in the concentration ranges used. 


\section{INTRODUCCIÓN}

Los ácidos orgánicos, los azúcares y los compuestos fenólicos son las moléculas de mayor presencia en los jugos de frutas. Evaluar estas sustancias en los alimentos es importante dado que afectan las propiedades sensoriales e influyen en la estabilidad de los productos terminados (Kelebek,2009).Asimismo, enlosjugosylosnéctareslosácidos evidencian la autenticidad del producto y son usados con este fin por su baja susceptibilidad al cambio durante los procesos de manufactura y almacenamiento (Reuter, 2015; Cunha et al., 2002). Los ácidos más usados como aditivos para mejorar las calidades sensoriales de las bebidas son el ácido cítrico, el tartárico, el fumárico y el fosfórico. Entre tanto, el ácido benzoico es empleado como preservante para disminuir el $\mathrm{pH}$ del jugo y con esto aumentar el tiempo de vida útil en estante de las bebidas comerciales (Shui y Leong, 2002).

Diversas técnicas analíticas han sido empleadas para la cualificación y la cuantificación de ácidos orgánicos en alimentos. Sin embargo, las técnicas cromatográficas son las mejores por su simpleza, rapidez y reproducibilidad. Dentro de estas la más utilizada es la cromatografía líquida de alta eficiencia (CLAE), que requiere un mínimo de preparación de la muestra y emplea diversidad de fases estacionarias y de sistemas de detección, lo cual permite obtener resultados rápidos y precisos (Mato, 2005).

En Colombia las bebidas no alcohólicas mantienen una demanda creciente, en particular las alternativas más saludables como el agua embotellada, el té, los jugos y los concentrados de frutas, que han desplazado a las bebidas tradicionales como las gaseosas (Legiscomex, 2014). Para las bebidas con adición de jugo (zumo) o pulpa de fruta, o las mezclas de estos que se distribuyen en Colombia, el Ministerio de Salud y Protección Social (2013) elaboró un reglamento técnico en el cual se estipulan los requisitos mínimos que deben cumplir para su comercialización nacional.

El propósito de este trabajo fue separar, identificar y cuantificar los ácidos orgánicos presentes en bebidas de fruta comerciales ampliamente consumidas por la población colombiana. Para ello se empleó cromatografía líquida de alta eficiencia con detección ultravioleta. De esa manera se verificó la calidad de estos productos consumidos en Colombia.

\section{MATERIALES Y MÉTODOS}

Los ácidos ascórbico, cítrico, málico, fórmico, succínico, tartárico y sulfúrico fueron Merck (Darmstadt, Alemania). El agua $\left(\mathrm{H}_{2} \mathrm{O}\right)$ desionizada se obtuvo en un equipo E-pure Barnstead (Thermo, Barnestead Lab, CA, Estados Unidos). Las soluciones patrón —de $1000 \mathrm{mg} \mathrm{L}^{-1}$ de los ácidos ascórbico, málico, fórmico, succínico, tartárico, y $2000 \mathrm{mg} \mathrm{L}^{-1}$ del ácido cítrico- fueron preparadas al disolver los estándares puros con la fase móvil empleada. Previo al análisis todas las soluciones fueron filtradas a través de membranas de 0,45 $\mu \mathrm{m}$ de poro (Millipore).
Para el análisis se empleó un cromatógrafo líquido UltiMate 3000, equipado con un sistema de procesamiento de datos Chromeleon 7.2, inyector automático con loop de $20 \mu \mathrm{L}$, un detector de longitud de onda fija a $210 \mathrm{~nm}$ y una columna de ODS de $5 \mu \mathrm{m}$ (250x4,6 mm). La fase móvil empleada fue ácido sulfúrico $1 \mathrm{mM}$ a un flujo de $1 \mathrm{~mL} \mathrm{~min}^{-1}$ y el tiempo total de cada corrida fue de 10 minutos. La fase móvil empleada se determinó realizando pruebas basadas en los trabajos de Karadeniz (2004) y Nour et al. (2010), que realizaron corridas cromatográficas en modo isocrático empleando ácido sulfúrico y ácido fosfórico a flujos entre $0,5 \mathrm{~mL} \mathrm{~min}^{-1}$ y $1 \mathrm{~mL} \mathrm{~min}$. .

Las muestras de bebidas (Tabla 1) fueron obtenidas de supermercados locales y almacenadas en nevera a $4{ }^{\circ} \mathrm{C}$ hasta su análisis. Para la evaluación $1 \mathrm{~mL}$ de la bebida comercial se diluyó en $4 \mathrm{~mL}$ de agua desionizada, mezcla que a continuación se centrifugó durante 10 min a 5000 rpm. Posteriormente, se tomaron $2 \mathrm{~mL}$ del sobrenadante y se filtraron a través de una membrana de nailon de 0,2 $\mu \mathrm{m}$ para ser finalmente sometidos al análisis (Karadeniz, 2004). Todas las muestras después de preparadas fueron inmediatamente corridas en el HPLC.

La identificación de los ácidos orgánicos fue realizada por comparación de los tiempos de retención de los estándares y de las muestras. Además, se adicionaron cantidades conocidas de los ácidos detectados a las muestras para verificar la identidad del pico. La cuantificación se realizó mediante curvas de calibración, que fueron construidas con seis niveles de concentración para el ácido ascórbico y siete niveles para el ácido cítrico. Todos los análisis se realizaron por triplicado (Fig. 1).

\section{RESULTADOS Y DISCUSIÓN}

Para optimizar las condiciones cromatográficas se evaluaron diferentes fases móviles, en las cuales se usaron tanto ácido sulfúrico como fosfórico, que son comúnmente reportados para la cuantificación de ácidos orgánicos por Clae (De Quirós et al., 2009; Chinnici et al., 2005; Pérez-Ruiz et al., 2004; De Villiers et al., 2004). La mejor separación se obtuvo al emplear la fase móvil isocrática compuesta por ácido sulfúrico

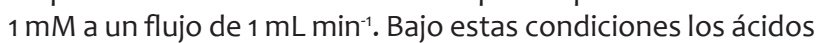
analizados tuvieron buena separación. Un cromatograma de la mezcla de los ácidos evaluados bajo las condiciones cromatográficas descritas se presenta en la Fig. 2.

Los análisis tuvieron buena repetibilidad por día $(n=3)$ y una apropiada precisión entre días $(n=10)$ respecto de los tiempos de retención con valores de $0,3 \%$ y 1,6\% de desviación estándar relativa (RSD), respectivamente. En cuanto al área del pico, la repetibilidad $(n=3)$ y la precisión $(n=3)$ estuvieron dentro del $5 \%$ de RSD. El porcentaje de recuperación del método se obtuvo al analizar una muestra preparada mediante la adición de cantidades conocidas de ácidos ascórbico o cítrico a una bebida. Las recuperaciones de los compuestos de interés estuvieron entre el $85 \%$ y el $106 \%$. El límite de detección (CMD, $\mathrm{S} / \mathrm{n}=3$ ) y el de cuantificación ( $C M C, \mathrm{~S} / \mathrm{n}=10$ ) de los ácidos cítrico y ascórbico a $210 \mathrm{~nm}$ se presentan en la Tabla 2 . 
Tabla 1. Muestras de bebidas analizadas.

\begin{tabular}{ccc}
\hline Muestra & Sabor & Ingredientes reportados en la etiqueta \\
\hline A1 & Coco & Agua, azúcar, ácido cítrico, ácido ascórbico, benzoato de sodio. \\
A2 & Granadilla & Agua, azúcar, ácido cítrico, ácido ascórbico, citrato de sodio. \\
A3 & Limón & Agua, azúcar, ácido cítrico, ácido ascórbico, benzoato de sodio. \\
B1 & Manzana & Agua, azúcar, ácido cítrico, citrato de sodio. \\
C1 & Mandarina & Agua, azúcar, ácido cítrico, ácido ascórbico, benzoato de sodio. \\
C2 & Naranja & Agua, azúcar, ácido cítrico, ácido ascórbico, benzoato de sodio. \\
D1 & Naranja & Agua, azúcar, ácido cítrico, ácido ascórbico, ácido málico, tartrazina. \\
E1 & Naranja & Agua, azúcar, ácido cítrico, ácido ascórbico, benzoato de sodio, tartrazina. \\
F1 & Mango & Agua, azúcar, ácido cítrico, ácido ascórbico. \\
F2 & Piña-naranja & Agua, azúcar, ácido cítrico, ácido ascórbico, citrato de sodio. \\
\hline
\end{tabular}
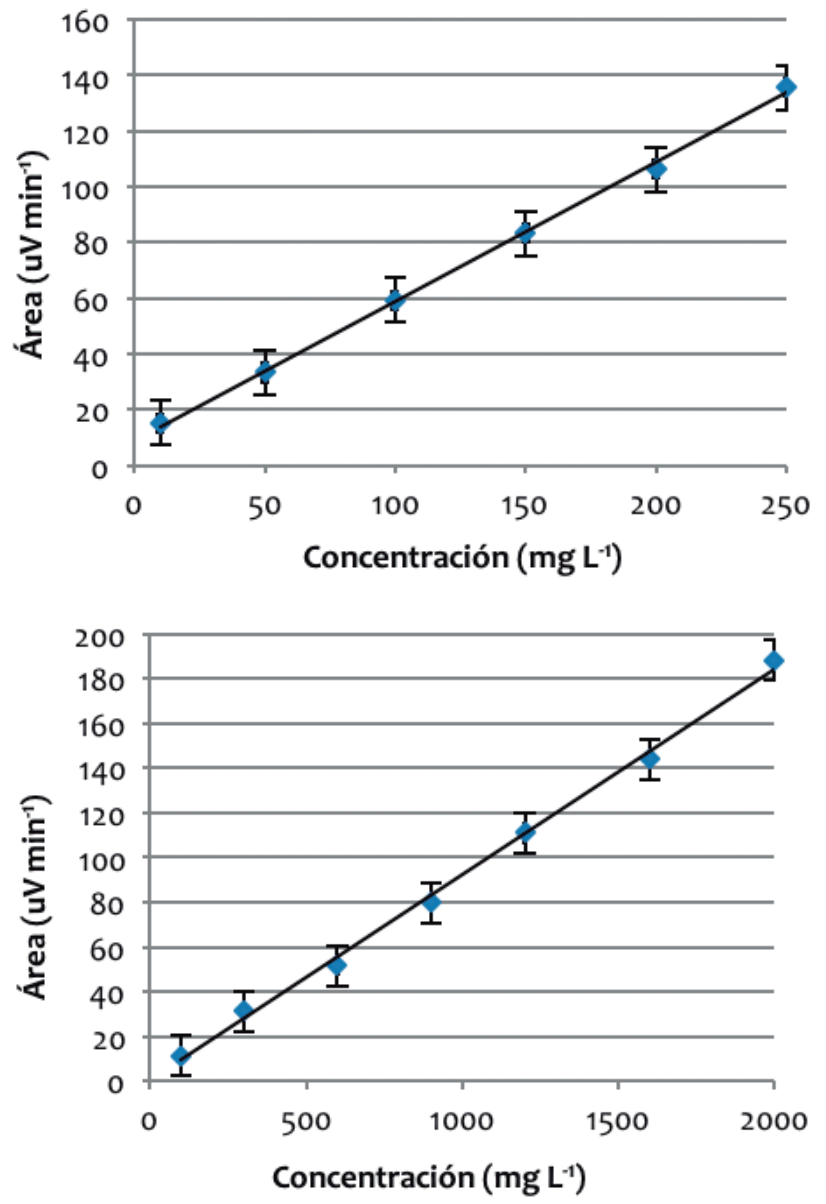

Figura 1. Rectas de calibración obtenidas para los ácidos: a) ascórbico y b) cítrico.

Bajo las condiciones cromatográficas empleadas el único ácido que se detectó en todas las bebidas evaluadas fue el cítrico (Fig. 2), y en cinco de ellas el ascórbico. Estos, junto con el ácido málico y el tartárico, son los que han sido reportados en mayor abundancia en las bebidas de frutas (Scherer et al., 2012). Estos resultados coinciden con lo reportado por Dias et al. (2014), que evaluaron treinta bebidas de frutas y detectaron en todas la presencia del ácido cítrico, mientras que el ascórbico estuvo presente en veintiocho muestras y el ácido tartárico no fue detectado. Aunque los ácidos málico y tartárico se encuentran en una gran cantidad de frutas, pueden no presentarse en alta concentración, lo cual hace difícil su detección en algunas muestras, como es el caso de las bebidas de frutas.

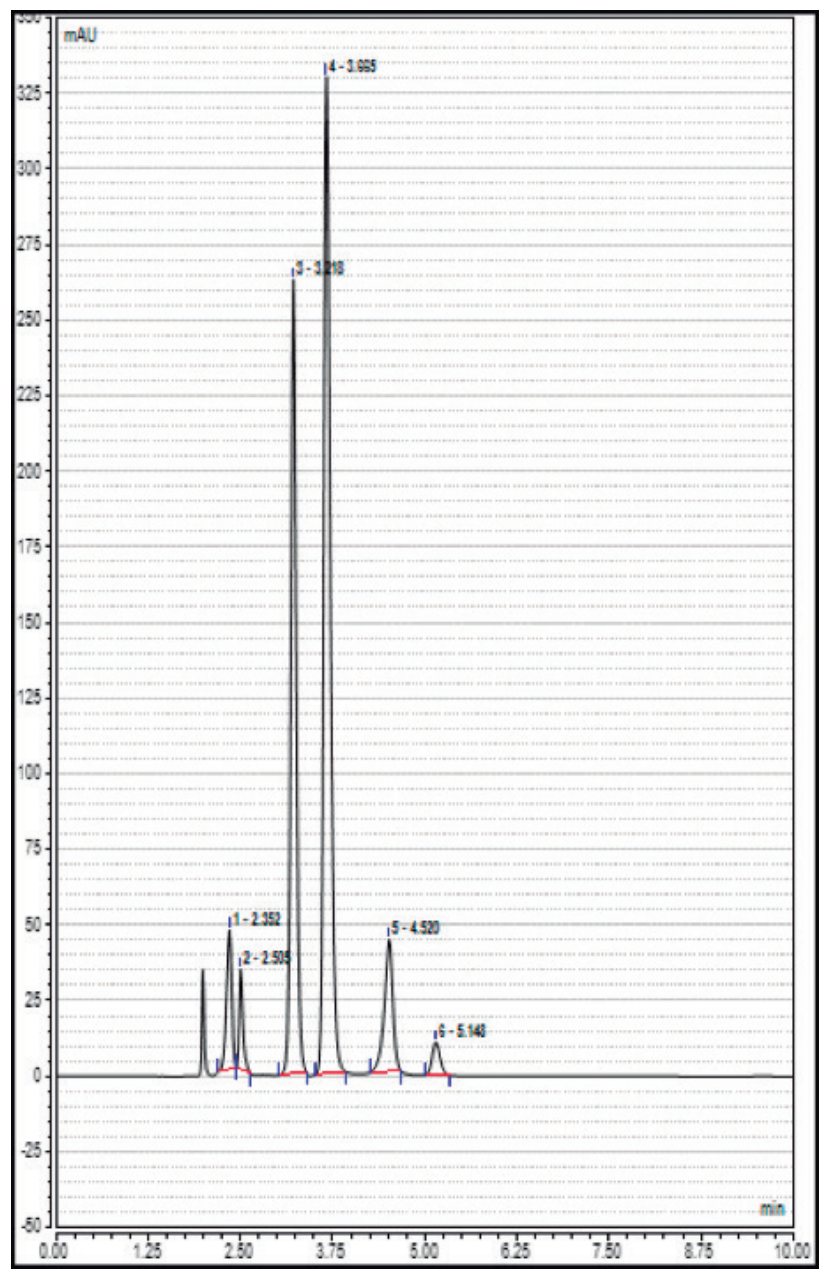

Figura 2. Cromatograma de la mezcla de estándares a $100 \mathrm{mg}$ L-1 de los ácidos: 1) tartárico, 2) fórmico, 3) ascórbico, 4) málico, 5) cítrico, 6) succínico. 
Tabla 2. Parámetros de las curvas de calibración y límites de cuantificación (CMC) y de detección (CMD) para los ácidos ascórbico y cítrico.

\begin{tabular}{|c|c|c|c|c|c|c|c|}
\hline Compuesto & $\begin{array}{l}\text { Tiempo de retención } \\
(\mathrm{min})\end{array}$ & $\begin{array}{c}\text { Intervalo lineal de } \\
\text { concentración }\left(\mathrm{mg} \mathrm{L}^{-1}\right)\end{array}$ & $\beta_{0}$ & $\beta_{1}$ & $\mathbf{r}^{2}$ & $\begin{array}{c}\mathrm{CMC} \\
\left(\mathrm{mg} \mathrm{L}^{-1}\right)\end{array}$ & $\begin{array}{l}\mathrm{CMD} \\
\left(\mathrm{mg} \mathrm{L}^{-1}\right)\end{array}$ \\
\hline Ácido ascórbico & $3,218 \pm 0,0830$ & $10-250$ & 9,191 & 0,4978 & 0,9989 & 6,67 & 2 \\
\hline Ácido cítrico & $4,520 \pm 0,1158$ & $100-2000$ & 0,221 & 0,0919 & 0,9972 & 83,33 & 25 \\
\hline
\end{tabular}

ßo: intercepto; $\beta 1$ : pendiente; r2: coeficiente de determinación

Tabla 3. Concentraciones del ácido ascórbico (AA) y el cítrico (AC) en las muestras de bebidas comerciales.

\begin{tabular}{cccc}
\hline \multirow{2}{*}{ Muestra } & Sabor & \multicolumn{2}{c}{ Concentración ( \pm desviación estándar) de ácidos (mg L-1) } \\
\cline { 3 - 4 } A1 & Coco & Ascórbico & $2816,3 \pm 124,1$ \\
A2 & Granadilla & $28,4 \pm 3,3$ & $2522,7 \pm 39,5$ \\
A3 & Limón & ND & $4476,7 \pm 293,3$ \\
B1 & Manzana & $28,4 \pm 15,0$ & $375,3 \pm 12,3$ \\
C1 & Mandarina & ND & $525,1 \pm 5,0$ \\
C2 & Naranja & $406,4 \pm 0,5$ & $1985,8 \pm 12,9$ \\
D1 & Naranja & ND & $3833,5 \pm 116,2$ \\
E1 & Naranja & $749,0 \pm 27,1$ & $5073,7 \pm 40,2$ \\
F1 & Mango & ND & $3025,8 \pm 22,6$ \\
F2 & Piña-naranja & ND & $2436,7 \pm 75,7$ \\
\hline
\end{tabular}

$\mathrm{ND}=$ no detectado

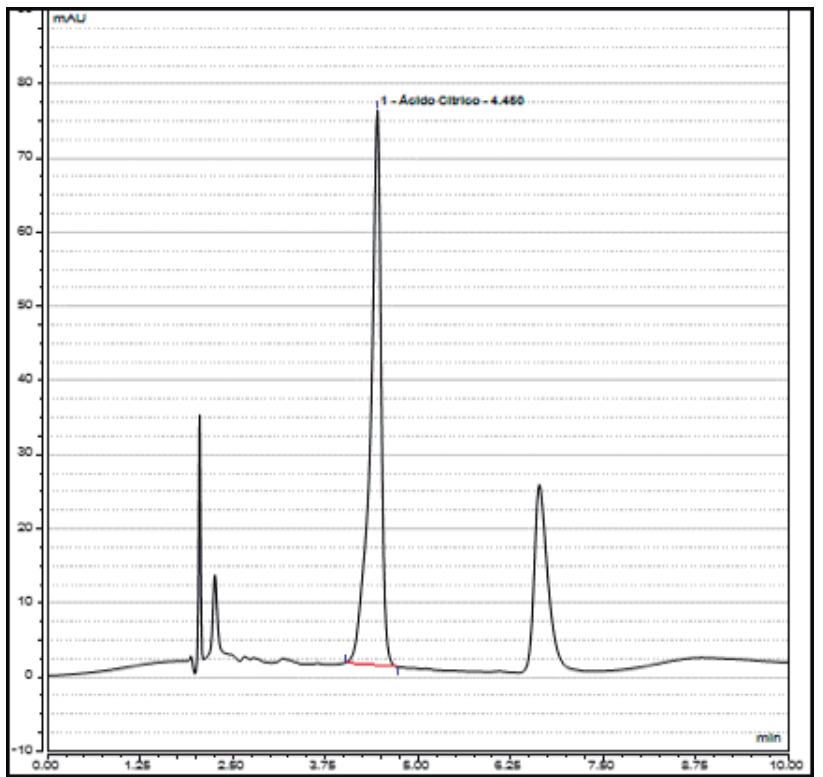

Figura 3. Cromatograma de una muestra de refresco comercial C2.

Por otro lado, la ausencia de los ácidos fórmico y succínico presupone buenas prácticas de manufactura dado que estos ácidos son producidos durante procesos de fermentación de cierto tipo de bebidas alcohólicas, como el vino (Özcelik et al., 2016), lo que no ocurre en las muestras analizadas en este trabajo. Sin embargo, la ausencia del ácido málico en la bebida de manzana no es un resultado acorde con los reportes bibliográficos, en los cuales se especifica que este es el ácido predominante en dicha fruta, con valores por encima del $90 \%$ del total de los ácidos orgánicos presentes (Wu et al., 2007; Zhang et al., 2010). Las concentraciones encontradas en cada una de las muestras evaluadas se presentan en la Tabla 3.
Como se puede observar en la Tabla 3, el ácido mayoritario en las bebidas de fruta analizadas fue el cítrico, que presentó una concentración entre 375,3 y $5073,7 \mathrm{mg} \mathrm{L}^{-1}$. El menor valor de dicho ácido se halló en la bebida de manzana (B1) y el mayor, en una de las de naranja (E1), lo cual es de esperarse dado que este ácido es el de mayor presencia en las frutas cítricas (Bermejo y Cano, 2012; Liu et al., 2012), y además es el que más se suministra a las bebidas de frutas para resaltar su sabor (Reuter, 2015). Esos datos también están de acuerdo con lo reportado por Dias et al. (2014), que obtuvieron concentraciones de ácido cítrico entre 1740 y $3740 \mathrm{mg} \mathrm{L}^{-1}$ para bebidas de naranja. Asimismo, se evidenció la presencia del ácido ascórbico en algunas de las muestras analizadas ( $A 1, A_{3}, C_{1}, D_{1}$ y F2), y las bebidas cítricas fueron las de mayor concentración de este ácido: naranja (D1), con 749,0 mg L-1, y mandarina (C1), con 406,4 mg L-1. Sin embargo, los resultados no son acordes con lo reportado por Bermejo y Cano (2012), quienes determinaron que este ácido era el más abundante en el limón que en la naranja. Vale la pena resaltar que la determinación de esta molécula tuvo mayor dificultad que la del ácido cítrico dado que el resultado variaba cuando el análisis se realizaba tiempo después de preparada la muestra. Esto coincide con lo reportado por Kabasakalis et al. (2000), quienes encontraron que en jugos comerciales la estabilidad de este ácido estaba relacionada con el tiempo y la temperatura de almacenamiento.

En cuanto a las concentraciones detectadas de los ácidos ascórbico y cítrico, se presentó alta variabilidad entre bebidas de diferente fruta, lo cual es esperado y concuerda con lo reportado en la Resolución 3929 de 2013 del Ministerio de Salud y Protección Social, y con los resultados de Bermejo y Cano (2012) y Liu et al. (2012), que evaluaron diferentes frutas por su contenido nutricional. También se evidenció alta variabilidad en productos del mismo origen. Sin embargo, es difícil hacer comparaciones dado que el perfil de los ácidos orgánicos y la concentración de estos en las frutas y los 
vegetales depende de factores como las especies, los suelos y las condiciones de estrés a las que se someten los cultivos de frutas (Bermejo y Cano, 2012). Asimismo, los diferentes protocolos de manufactura de las bebidas hacen complicada una posible comparación (Dias et al., 2014). No obstante, los valores obtenidos en todas las bebidas de frutas evaluadas, excepto las cítricas (limón, mandarina y naranja), están de acuerdo con lo estipulado en la norma general del códex para zumos (jugos) y néctares de frutas (Códex Stan 2472005), en la cual se indica que en jugos de frutas se permite una concentración de ácido cítrico de hasta $3000 \mathrm{mg} \mathrm{L}^{-1}$. La normativa colombiana para jugos, por su parte, no fija criterios ni límites específicos de aceptación o rechazo para los ácidos orgánicos presentes en las bebidas de frutas (Ministerio de Salud y Protección Social, 2013).

\section{CONCLUSIONES}

Diez bebidas comerciales obtenidas a partir de ocho tipos de frutas diferentes fueron analizadas por cromatografía líquida de alta eficiencia en este trabajo. Se encontró alta variabilidad en la concentración de los ácidos cítrico y ascórbico, que son los más abundantes en este tipo de refrescos.

La metodología cromatográfica empleada permitió el análisis rápido de seis ácidos orgánicos comúnmente encontrados en frutas. El método desarrollado mostró buena repetibilidad, reproducibilidad y linealidad, lo cual permite aseverar que fue apropiado y podría ser empleado en alimentos que contengan ese tipo de moléculas.

Los resultados obtenidos permiten concluir que las bebidas de frutas comercializadas en Colombia cumplen con los parámetros de calidad y salubridad requeridos en alimentos de alto consumo. Asimismo, se demostró que en el proceso de manufactura cada empresa emplea sus propias formulaciones, lo cual explica las diferencias encontradas entre las distintas bebidas analizadas.

\section{AGRADECIMIENTOS}

Los autores agraden a la Universidad de Caldas por el apoyo a la investigación.

\section{REFERENCIAS}

- Bermejo A y Cano A. 2012. Analysis of nutritional constituents in twenty citrus cultivars from the Mediterranean area at different stages of ripening. Food and Nutrition Sciences, 3:639-650.

- Chinnici F, Spinabelli U, Riponi C y Amati A. 2005. Optimization of the determination of organic acids and sugars in fruit juices by ion-exclusion liquid chromatography. Journal of Food Composition and Analysis, 18:121-130.

- Codex Stan 247. 2005. Norma general del códex para zumos (jugos) y néctares de frutas. 21 p. file:///C:/Users/PC/Downloads/CXS 247s.pdf, consultado septiembre de 2018 .
- Cunha SC, Fernandes JO y Ferriera I. 2002. HPLC/UV determination of organic acids in fruit juices and nectars. European Food Research and Technology, 214:67-71.

- De Quirós A, Lage-Yusty MA y López-Hernández J. 2009. HPLC analysis of organic acids using a novel stationary phase. Talanta, 78:643-646.

- De Villiers A, Lynen F, Crouch A y Sandra P. 2004. Development of a solid-phase extraction procedure for the simultaneous determination of polyphenols, organic acids and sugars in wine. Chromatography, 59:403-409.

- Dias LG, Sequeira C, Veloso ACA, Morais JS, Sousa M y Peres AM. 2014. A size exclusión HPLC method for evaluating the individual impacts of sugars and organic acids on beverage global taste by means of calculate dose-overthreshold values. Chromatography, 1:141-158.

- Kabasakalis V, Siopidou D y Moshatou E. 2000. Ascorbic acid content of commercial fruit juices and its rate of loss upon storage. Food Chemistry, 70:325-328.

- Karadeniz F. 2004. Main organic acid distribution of authentic citrus juices inTurkey, Turk. Turkish Journal of Agriculture and Forestry, 28:267-271.

- Kelebek H, Selli S, Canbas A y Cabaroglu T. 2009. HPLC determination of organic acids, sugars, phenolic compositions and antioxidant capacity of orange juice and orange wine made from a Turkish cv. Kozan Microchemical Journal, 91:187-192.

- Legiscomex. 2014. Bebidas no alcohólicas en Colombia/informe sectorial. https://goo.gl/fZZZBU, consulta abril de 2017.

- Liu Y, Heying E y Tanumihardjo A. 2012. History, global distribution and nutritional importance of citrus fruits: Comprehensive reviews. Comprehensive Reviews in Food Sciences and Food Safety, 11:534-535.

- Mato I, Suárez-Luque S y Huidobro JF. 2005. A review of the analytical methods to determine organic acids in grape juices and wines. Food Research International, 38, 1175-1188.

- Ministerio de Salud y Protección Social. 2013. Resolución 3929. Reglamento técnico sobre los requisitos sanitarios que deben cumplir las frutas y las bebidas con adición de jugo (zumo) o pulpa de fruta o concentrados de fruta, clarificados o no, o las mezclas de estos que se procesen, empaquen, transporten, importen y comercialicen en el territorio nacional. https://goo. gl/6nHuN9, consultado septiembre de 2018.

- Nour V, Trandafir I y lonica ME. 2010. HPLC organic acid analysis in different citrus juices under reversed phase conditions. Notulae Botanicae Horti Agrobotanici Cluj-Napoca, 38:44-48.

- Özcelik S, Kuley E y Ozogul F. 2016. Formation of lactic, acetic, succinic, propionic, formic and butyric acid by lactic acid bacteria. Food Science and Technology, 73:536-542.

- Pérez-Ruiz T, Martínez-Lozano C, Tomás V y Martín J. (2004). Highperformance liquid chromatographic separation and quantification of citric, lactic, malic, oxalic and tartaric acids using a post-column photochemical reaction and chemiluminescence detection. Journal of Chromatography $A$, 1026:57-64.

- Reuter, W. 2015. Analysis of organic acids in fruit juices by HPLC and UV detection. Application note, PerkinElmer.

- Scherer R, Poloni AC, Ballus CA, Dillenburg A, Teixeira J y Teixeira H. 2012. Validation of a HPLC method for simultaneous determination of main organic acids in fruits and juices. Food Chemistry, 135:150-154.

- Shui G y Leong LP. 2002. Separation and determination of organic acids and phenolic compounds in fruit juices and drinks by high-performance liquid chromatography. Journal of Chromatography A, 977(1): 89-96.

- Wu J, Gao H, Zhao L, Liao X, Chen F, Wang Z y Hu X. 2007. Chemical compositional characterization of some apple cultivars. Food Chemistry, 103:88-93.

- Zhang Y, Li P y Cheng L. 2010. Developmental changes of carbohydrates, organic acids, amino acids, and phenolic compounds in "Honeycrisp" apple flesh. Food Chemistry, 123:1013-1018. 